\title{
Host Plant Resistance to Tomato spotted wilt virus (Bunyaviridae: Tospovirus) in Tomato
}

\author{
David G. Riley ${ }^{1}$ and Shimat V. Joseph \\ Department of Entomology, University of Georgia, Tifton Campus, $122 \mathrm{~S}$. \\ Entomology Drive, Tifton, GA 31793 \\ W. Terry Kelley \\ Harris Moran Seed Company, P.O. Box 4938, Modesto, CA 95352
}

Steve Olson and John Scott

Horticultural Sciences Department, 1117 Fifield Hall, P.O. Box 110690, Gainesville, FL 32611-0690

Additional index words. Solanum lycopersicum, Thripidae, Frankliniella fusca

\begin{abstract}
Commercially available cultivars of tomato Solanum lycopersicum L. were fieldtested for resistance to Tomato spotted wilt virus (TSWV) over a 5-year period (from 2006 to 2010) at the Coastal Plain Experiment Station at Tifton, GA. Selected cultivars were transplanted each year into staked, black plastic mulch beds on drip irrigation in the spring of each year when the incidence of Tomato spotted wilt (TSW) tended to be highest. The presence of TSWV was confirmed by double antibody sandwich (DAS) enzyme-linked immunosorbent assay (ELISA). Also, the presence of thrips vectors was monitored using beat-cup sampling of foliage and flower samples. Tomato cultivars with the $S w$ 5 resistance gene provided high levels of control of TSW expression over all 5 years. However, these genotypes had no apparent effect on the thrips vectors, western flower thrips, Frankliniella occidentalis (Pergande), and tobacco thrips, Frankliniella fusca (Hinds), that transmit TSWV. Overall, the top 15 commercial tomato cultivars based on consistent TSW resistance and ranked from highest marketable fruit yield were: 'Tycoon', 'Tous 91', 'Talladega', 'Red Defender', 'BHN 444', 'Nico', 'Carson', 'BHN 685 (Roma type)', 'Picus', 'Redline', 'Tribute', 'Quincy', 'BHN 640', 'BHN 602', and 'Top Gun'.
\end{abstract}

Thrips-transmitted TSWV (Tospovirus: Bunyaviridae) has caused serious losses to tomato, Solanum lycopersicum L., production worldwide (Goldbach and Peters, 1994, Persley et al., 2006). In Georgia alone, the losses to tomato and pepper combined resulting from TSW was $\$ 90$ million from 1997 to 2006. TSWV is known to induce a wide range of symptoms, including reddish brown ringspots on foliage or interveinal speckling of the foliage that later coalesce and turn into necrotic area (Best, 1968; Gitaitis, 2009). If the infection is early, plants can be severely stunted or show severe wilt stress. Tomato fruit generally displays a yellow ringspot symptom in red ripe fruit or there can be raised ringspots and bronzing of tissue on mature green fruit (Olson, 2009). Two main vectors of TSW in Georgia are western flower thrips, Frankliniella occidentalis (Pergande), and tobacco thrips, Frankliniella fusca (Hinds) (Riley and Pappu, 2000, 2004).

Normally, immature thrips feed on TSWVinfected weed plants surrounding vegetable fields, acquire the virus, and move into tomato

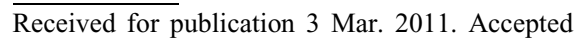
for publication 5 May 2011.

${ }^{1}$ To whom reprint requests should be addressed; e-maildgr@uga.edu. from L. chilense (Stevens et al., 2006), or both have been selected for introgression to commercial lines, which are being marketed as resistant cultivars. The $S w-5$ gene is reported to produce a hypersensitive response in the tomato foliage, which effectively restricts spread of TSW on infection (Aramburu and Rodriguez, 1999). Cultivars that possess the $S w-5$ gene have provided high levels of resistance to TSWV isolates in greenhouse and field studies (Boiteux and de Giordano, 1993; Díez et al., 1995; Mandal et al., 2006; Mitidieri et al., 2001). However, a few TSWV isolates, TSWV6, $\mathrm{An}_{\mathrm{wa}}-1, \mathrm{Da}_{\mathrm{WA}}-1 \mathrm{~d}$, and $\mathrm{TO}_{\mathrm{TAS}}-1 \mathrm{~d}$, were reported to overcome the resistance provided by $\mathrm{Sw}-5$ gene (Aramburu and Marti, 2003; Ciuffo et al., 2005; Gordillo et al., 2008; Latham and Jones, 1998). These resistant breaking isolates have intensified research to find additional reliable genes from wild relatives of cultivated tomato (Canady et al., 2001; Pico et al., 2002). A recently identified gene adapted to the southeastern United States, $S w-7$, is effective against TSWV6 and $\mathrm{An}_{\mathrm{wa}}-1$ isolates and is being introgressed to commercial lines (Saidi and Warade, 2008; Stevens et al., 2006).

Most commercially available TSW-resistant tomato cultivars carry the $S w-5$ gene (Table 1). However, in the southeastern United States, published information related to marketable tomato yield among TSW-resistant cultivars under varying disease incidence is still lacking. Riley and Pappu $(2000,2004)$ provided the only published data on possible effects of tomato cultivars on thrips populations, but only two cultivars were compared at a time. Therefore, the objective of the present study was to assess: the level of resistance to TSW, the incidence of thrips vectors, and the relative tomato yield among multiple commercial and experimental TSW-resistant tomato cultivars under the natural incidence of TSW over multiple years. We specifically tested tomato cultivars for their ability to influence thrips populations under field conditions. Our hypothesis was that the resistance to TSW currently available in commercial tomato is only related to suppression of virus symptom expression and not related to effects on thrips vector populations in the field.

\section{Materials and Methods} ticide treatments (Brown and Brown, 1992; Riley and Pappu, 2000), host-plant resistance (Krishna et al., 1993; Kumar et al., 1995), and combinations of tactics for early-season management of thrips vector populations (Cho et al., 1989; Riley and Pappu, 2004). Above all, development of TSW-resistant cultivars has been identified as the most sustainable solution to reduce TSW incidence in tomato (de Haan et al., 1996; Gordillo et al., 2008; Iizuka et al., 1993; Riley and Pappu, 2004; Saidi and Warade, 2008).

Several accessions closely related to $\mathrm{Lyco}$ persicum have been evaluated and relatively few promising genes that induce resistance have been identified (reviewed by Saidi and Warade, 2008). Among the thoroughly studied genes, $S w-5$ from L. peruvianum (Stevens et al., 1992, 1994, 1995), a new gene, $S w-7$
Plant materials, field design, and management. The field studies were conducted each spring in 2006-2010 at the Coastal Plain Experiment Station, Tifton, GA, to simultaneously evaluate different TSW-resistant tomato cultivars unprotected from thrips vectors (Table 1). A randomized complete block design with four replicates was used each year except in 2006 when there were three replications. The tomato production system used was raised, black plastic-covered beds fumigated with methyl bromide $(277 \mathrm{~kg}$ a.i./ha $98 \%$ ) with plants staked and tied at $0.46-\mathrm{m}$ spacing in a single 7-m length row per plot. Tomatoes were transplanted by year on 23 Mar., 4 Apr., 17 Apr., 19 Apr., and 30 Mar. for 2006, 2007, 2008, 2009, and 2010, respectively. 
Table 1. Tomato cultivars, their source, resistance designation, Tomato spotted wilt (TSW) resistance confirmation. and average plot yield for the years evaluated from 2006 to 2010.

\begin{tabular}{|c|c|c|c|c|}
\hline Cultivar $^{2}$ & Plant source & $\begin{array}{c}\text { TSW-resistant } \\
\text { gene }^{\mathrm{y}}\end{array}$ & $\begin{array}{l}\text { TSW resistance } \\
\text { expression }^{\mathrm{x}}\end{array}$ & $\begin{array}{c}\text { Marketable } \\
\text { fruit yield }(\mathrm{kg})\end{array}$ \\
\hline Quincy & Seminis & + & $* * * * *(5)$ & $9.1 \pm 1.0$ \\
\hline BHN 640 & BHN/Siegers & + & $* * * * *(5)$ & $9.0 \pm 0.9$ \\
\hline Crista & Harris Moran/Clifton & + & $* * * * *(5)$ & $8.6 \pm 1.2$ \\
\hline Bella Rosa & Sakata/Siegers & + & $* * * * *(5)$ & $7.9 \pm 0.9$ \\
\hline Amelia & Harris Moran/Clifton & + & $* * * * *(5)$ & $7.5 \pm 0.8$ \\
\hline Talladega & Syngenta & + & $* * * *(4)$ & $10.1 \pm 0.9$ \\
\hline Red Defender & Harris Moran & + & $* * * *(4)$ & $9.8 \pm 0.7$ \\
\hline BHN 444 & BHN/Siegers & + & $* * * *(4)$ & $9.7 \pm 0.6$ \\
\hline Redline & Syngenta & + & $* * * *(4)$ & $9.3 \pm 0.7$ \\
\hline BHN 602 & BHN/Siegers & + & $* * * *(4)$ & $8.9 \pm 0.8$ \\
\hline Top Gun & Twilley & + & $* * * *(4)$ & $8.8 \pm 0.7$ \\
\hline Inbar & Hazera & + & $* * * *$ (4) & $7.3 \pm 1.1$ \\
\hline Tycoon & Hazera & + & $* *(2)$ & $10.9 \pm 1.0$ \\
\hline Carson & Hazera & + & $* *(2)$ & $9.5 \pm 1.2$ \\
\hline Picus & Seminis & + & $* *(2)$ & $9.5 \pm 1.5$ \\
\hline BHN 685 & Siegers & + & $* *(2)$ & $9.5 \pm 1.2$ \\
\hline Shanty (Roma) & Hazera & + & $* *(2)$ & $8.7 \pm 0.8$ \\
\hline Tribeca & Vilmorin & + & $* *(2)$ & $8.7 \pm 0.9$ \\
\hline Mountain Glory & Siegers & + & $* *(2)$ & $8.4 \pm 0.9$ \\
\hline Fletcher & Reimer Seeds & + & $* *(2)$ & $8.0 \pm 0.4$ \\
\hline Muriel & Sakata/Siegers & + & $* *(2)$ & $7.8 \pm 1.0$ \\
\hline Finishline & Syngenta & + & $* *(2)$ & $7.7 \pm 0.8$ \\
\hline 8746 & UF & + & $*(1)$ & $13.2 \pm 0.2$ \\
\hline Tous 91 & Hazera & + & * (1) & $10.2 \pm 0.9$ \\
\hline 8751 & UF & $\wedge$ & * (1) & $9.7 \pm 1.7$ \\
\hline Nico & Harris Moran & + & $*(1)$ & $9.5 \pm 0.2$ \\
\hline 8612 & UF & + & * (1) & $9.4 \pm 1.1$ \\
\hline 8749 & UF & $+^{\wedge}$ & *(1) & $9.2 \pm 2.3$ \\
\hline Tribute & Sakata & + & $*(1)$ & $9.2 \pm 0.7$ \\
\hline HAZ 3089 & Hazera & + & *(1) & $8.8 \pm 0.9$ \\
\hline HAZ 3084 & Hazera & + & $*(1)$ & $8.6 \pm 0.6$ \\
\hline Rubia (Roma) & Sakata & + & $*(1)$ & $8.5 \pm 0.2$ \\
\hline 8768 & UF & + & * (1) & $8.4 \pm 1.2$ \\
\hline 8688 & UF & $+^{\wedge}$ & *(1) & $7.6 \pm 0.4$ \\
\hline 8793 & UF & $\wedge$ & * (1) & $7.5 \pm 1.2$ \\
\hline 8685 & UF & + & * (1) & $7.5 \pm 0.2$ \\
\hline HAZ 3088 & Hazera & + & $*(1)$ & $6.2 \pm 0.6$ \\
\hline FTM 2305 & Sakata & + & * (1) & $6.2 \pm 1.9$ \\
\hline 8684 & UF & + & *(1) & $6.0 \pm 0.6$ \\
\hline 8687 & UF & $\wedge$ & * (1) & $5.8 \pm 1.3$ \\
\hline 8686 & UF & $\wedge$ & * (1) & $4.8 \pm 0.3$ \\
\hline Hedvig & Hazera & + & $*(1)$ & $4.5 \pm 0.6$ \\
\hline Galilea & Hazera & + & $*(1)$ & $4.4 \pm 2.0$ \\
\hline Cupid & Seminis & - & $* * *(3)$ & $3.3 \pm 0.9$ \\
\hline SecuriTY 28 & Harris Moran & - & (3) & $6.6 \pm 1.4$ \\
\hline Big Boy & Ferry Morse & - & (1) & $6.1 \pm 0.6$ \\
\hline Tygress & Seminis & - & (3) & $5.9 \pm 0.9$ \\
\hline Mariana (Roma) & Sakata/Rupp & - & (3) & $5.4 \pm 1.2$ \\
\hline FL 47 & Seminis/Siegers & - & (5) & $4.9 \pm 0.9$ \\
\hline Caporal & Vilmorin/Clifton & - & (3) & $4.7 \pm 0.9$ \\
\hline Pony Express & Harris Moran & - & (1) & $4.6 \pm 1.5$ \\
\hline Marglobe & USDA & - & (2) & $2.8 \pm 1.1$ \\
\hline
\end{tabular}

${ }^{\mathrm{z}}$ Cultivars ranked (from highest to lowest) based on the marketable fruit yield (kg) within number of years they were tested.

$\mathrm{y}_{+}=\mathrm{Sw}-5 ; \wedge=\mathrm{Sw}-7$ present

${ }^{\mathrm{x}}$ Number of year(s) when significant TSW virus resistance was detected $(*)$ on a selected cultivar compared with susceptible cultivars per total years evaluated (in parentheses). Cultivars are arranged within expression group from highest to lowest yield.

A minimum of $560 \mathrm{~kg} \cdot \mathrm{ha}^{-1}$ of $10 \mathrm{~N}-4.4 \mathrm{P}-$ $8.3 \mathrm{~K}$ was applied to Tift pebbly clay loam field plots each year and liquid fertilizer $8 \mathrm{~kg} \cdot \mathrm{ha}^{-1}$ $(7 \mathrm{~N}-0 \mathrm{P}-5.8 \mathrm{~K})$ was applied every 2 weeks through a drip irrigation system. In these tests, tomatoes were treated weekly in April and May with a mefenoxam plus chlorothalonil and Bacillus thuriengensis subsp. kurstaki to prevent disease and reduce Lepidoptera damage without affecting thrips populations. Paraquat was applied to row middles for weed control.
Disease ratings and enzyme-linked immunosorbent assay. Tomato plants were monitored for TSW symptoms on foliage and fruits (Gitaitis, 2009). Disease ratings were done on 18,22 , and 30 May and 5 June in 2006; 2, 10 , 18, 22, and 29 May in 2007; 6, 14, 20, and 28 May and 2 and 18 June in 2008; 27 Apr., 11, 19, and 29 May, and 4, 10, and 19 June in 2009; and 12, 16, 23, and 28 Apr., 7, 11, and 25 May, and 10 and 21 June in 2010. The number of plants with foliar TSW disease symptoms per plot was recorded throughout the season and percent TSW incidence was calculated based on visible symptoms.

A single, fully expanded terminal leaflet was randomly collected from the top third of each of six plants after fruit set in 2006 from each plot to detect TSWV with DAS ELISA using a TSWV detection kit. A sample was deemed positive for TSWV if the absorbance reading was three times the value of a known uninfected sample. In 2009, and in 2010, 10 leaf samples were taken from individual plots before harvest to confirm the presence of TSWV using DAS ELISA.

Thrips samples and evaluation. For all 5 years, the total number of thrips by species was determined using a sample beat-cup and blossom samples. Beat-cup samples were collected on 2 May in 2006; 23 Apr. and 7 May in 2008; 29 Apr., 7 May, and 15 May in 2009; and 21 Apr. and 13 May in 2010, whereas blossom samples were collected on 5 June in 2008 and 22 and 29 May in 2009. For beat-cup samples, we adopted the procedure described by Joost and Riley (2004). The procedure for blossom samples were 10 blossoms per plot, one blossom per plant, and which was randomly collected and placed into a vial with a $50 \%$ ethanol solution per plot on a weekly basis. Adult thrips in the blossom samples were identified using identification keys (Oetting et al., 1993; Stannard, 1968) under $70 \times$ to $140 \times$ magnification using a SZH10 Olympus ${ }^{\circledR}$ (Olympus America, Lake Success, NY) stereomicroscope. Only $F$. occidentallis and $F$. fusca were individually counted and all other thrips, including $F$. tritici, F. bispinosa, and others, were placed into an "other" category. From all samples, a subset of thrips was slidemounted for voucher specimens. Key characters were used to verify species including the anteromarginal and anteroangular setae, postocular setae, the pedicel of the third antennal segment, comb on abdominal tergite VIII, and other features (Stannard, 1968).

Yield assessment. Yield was assessed on 6,15 , and 20 June in 2006; 20 and 28 June in 2007; 25 June and 8 July in 2008; 17 and 29 June and 13 July in 2009; and 8 and 22 June in 2010. Fruit samples were harvested from the center six plants per subplot by quantifying fruit in various damage categories and marketable categories by size at the time of harvest using USDA standards for fresh market tomato (Sargent and Moretti, 2004). The exception to this was the 2009 evaluation of Roma-type tomatoes in which the fruit were rated only as marketable (good shape, size, and no apparent damage) or unmarketable (insector disease-damaged). Thrips damage to the fruit consisted of dimpling of the fruit surface (Olson, 2009), Lepidoptera-damaged fruit, physiological fruit damage, blotchy-colored fruit, and blossom end rot resulted in fruit being counted as unmarketable. In 2007, great care was taken to count irregularly ripened or blotchy-colored fruit at the time of harvest, assumed to be the result of TSW because the other potential causes such as whiteflies (Bullock et al., 1998) were virtually absent (i.e., whiteflies were not observed on foliage). Also, most of the irregular ripening exhibited the ringspot 
patterns that were associated with TSW (Olson, 2009). Damaged or unmarketable yield was based on the condition of all fruit at the time of harvest. The 2007 data for TSW irregularly ripened fruit assessed at the time of harvest was not included in marketable fruit. However, in 2009, a subsample of clean marketable fruit was gassed with $100 \mathrm{ppm}$ of ethylene for $24 \mathrm{~h}$ and then held for 1 week to assess the potential for irregular ripening in fruit rigorously graded at harvest. For marketable yield, the approximate value of the crop was estimated per acre using $\$ 8.9, \$ 7.1, \$ 8.0, \$ 12.2$, and $\$ 12.4$ per $11.3-\mathrm{kg}$ carton of marketable fruit for 2006, 2007, 2008, 2009, and 2010, respectively (USDA National Agricultural Statistical Service) and a tomato plant population of 12,100 plants/ha.

Statistical analyses were conducted to determine if the independent variables such as marketable or unmarketable yield, TSW rating, or thrips have any interaction between cultivars and years tested. Both yield and the rank of yields (PROC RANK for nonparametric comparisons) of the six cultivars, which were evaluated in all 5 years, were analyzed using PROC GLM (SAS Institute, 2003) to test for cultivar-by-year interaction. Based on significant year-by-cultivar interaction for yield and TSW symptoms, analysis of variance was conducted by year using PROC GLM. Means were determined at the cultivar level using least significant difference tests. PROC CORR (SAS Institute, 2003) was used to provide a measure of the overall correlation of seasonal averages of thrips from Years 2006, 2008, 2009, and 2010 to yield and TSW. The number of years in which a resistant cultivar was confirmed with field evaluations is summarized in Table 1 along with average marketable yield over the number of years tested.

\section{Results and Discussion}

The commercially available TSW-resistant tomato cultivars consistently demonstrated a lack of TSW symptom expression over the 5 years tested (Table 1; Figs. 1-5). Surprisingly, even one susceptible line, Cupid, a grape-type tomato, also ranked low in foliar symptom expression, possibly as a result of the difficulty in observing clear TSW symptoms in this type of tomato leaf canopy with abundant small leaflets. Based on fruit symptom expression, Cupid is clearly a TSW-susceptible line (Fig. 2). The TSW intensity varied greatly over the 5 years with annual final incidence of TSW symptomatic plants in 'FL 47', the susceptible control cultivar, reaching 94\%, 15\%, 42\%, 99\% (Figs. 1, 3, 4, and 5, respectively), and 5\% at harvest for 2006-2010, respectively. An assessment of the year-by-cultivar interaction using the marketable yields and ranks of yield for the six cultivars evaluated over all 5 years (Table 1) revealed significant interactions $\left(F_{20,65}=3.67 ; P<0.0001\right.$ and $F_{20,65}=3.53$; $P<0.0001$, respectively). Thus, year-by-year analyses of variances were deemed necessary. Even so, the average yields of resistant cultivars over all tests were clearly greater (no

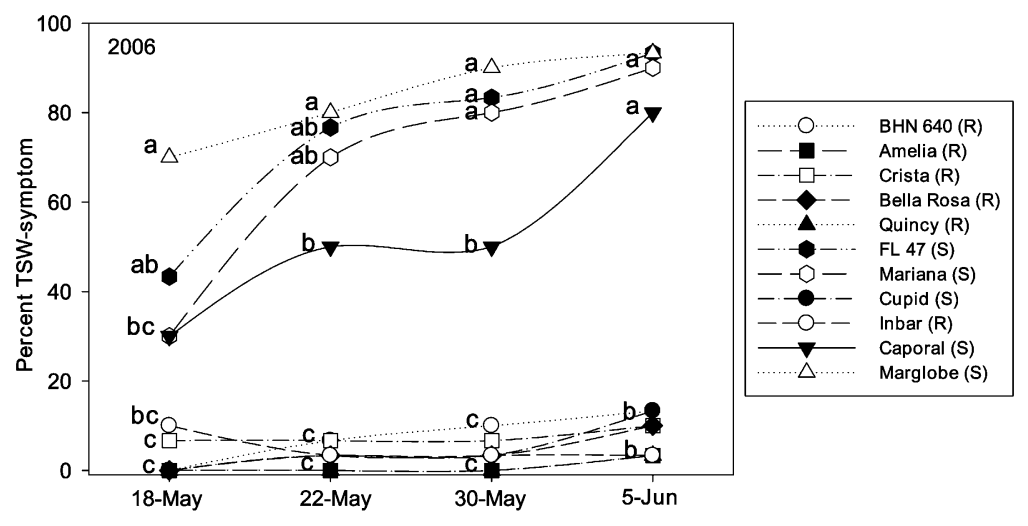

Fig. 1. The incidence of Tomato spotted wilt symptom in 2006. Means followed by the same letter within a sample date are not significantly different (least significant difference test, $P<0.05$ ).

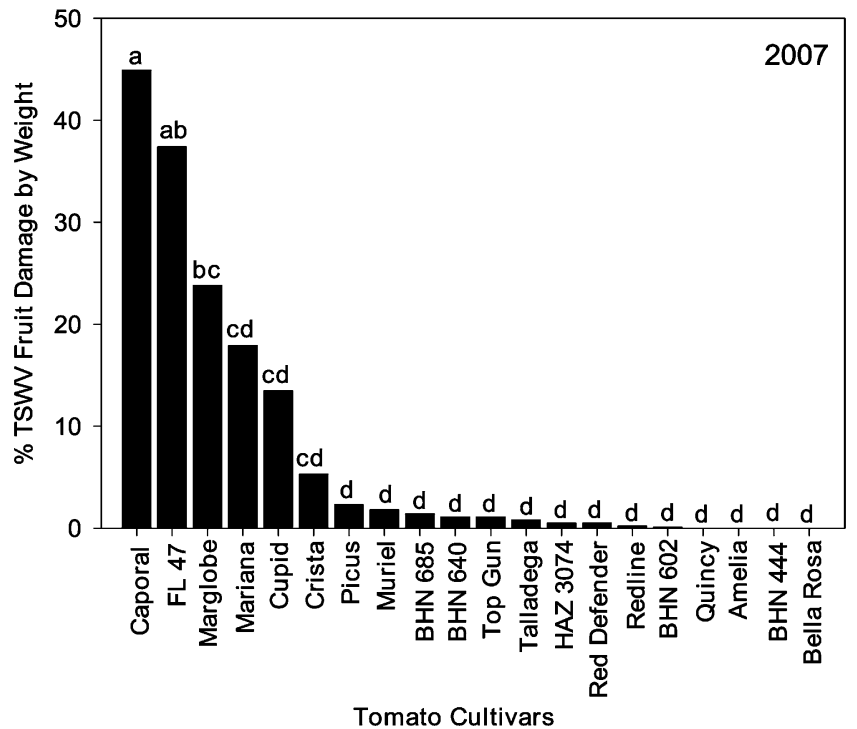

Fig. 2. Percent Tomato spotted wilt-damaged fruits by weight among cultivars in 2007 . Means followed by the same letter are not significantly different (least significant difference test, $P<0.05$ ).

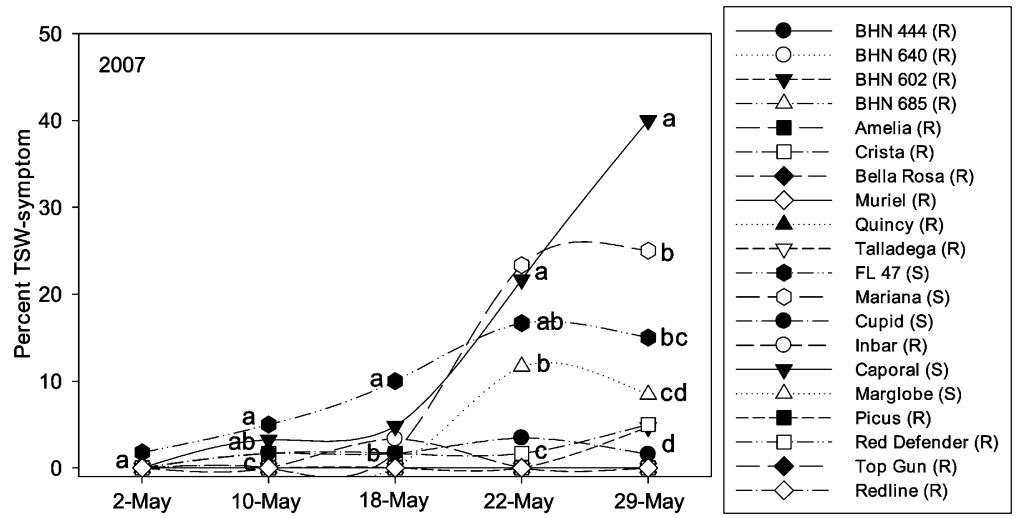

Fig. 3. The incidence of Tomato spotted wilt symptom in 2007. Means followed by the same letter within a sample date are not significantly different (least significant difference test, $P<0.05$ ).

overlapping SES) than the susceptible control, 'FL 47' (Table 1), indicating that in a TSW-prone tomato production region such as Georgia, TSW-resistant lines perform better than susceptible commercial lines in 4 of 5 years (Tables 2-6).
In 2006, TSW disease pressure was relatively high and the TSW symptoms were significantly more apparent in susceptible cultivars such as Marglobe, FL 47, Mariana, or Caporal than on those cultivars marketed as TSWresistant (Table 2). Percent TSW disease 


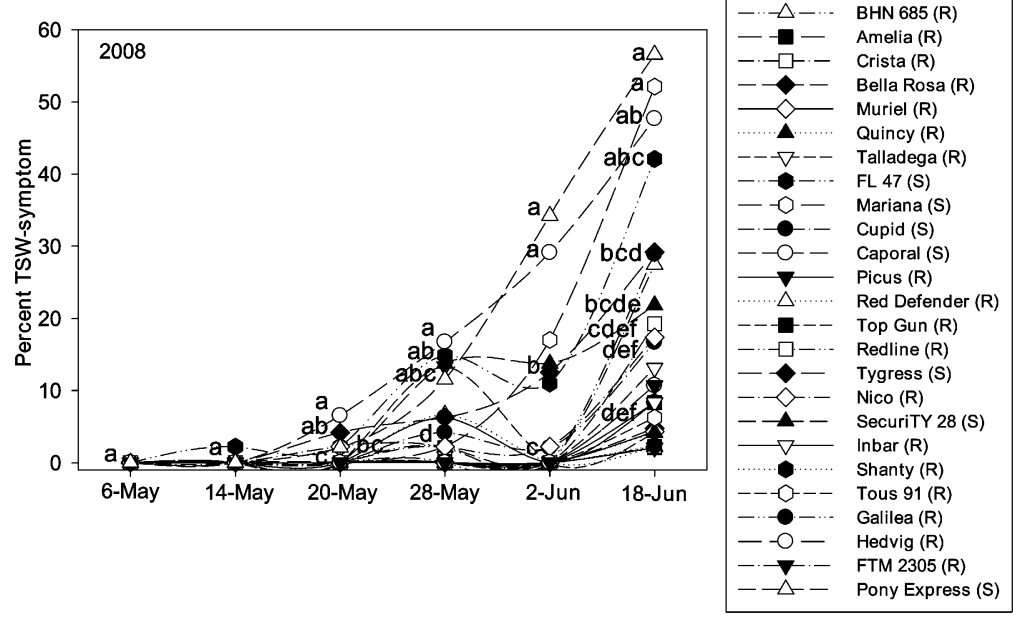

Fig. 4. The incidence of Tomato spotted wilt symptom in 2008. Means followed by the same letter within a sample date are not significantly different (least significant difference test, $P<0.05$ ).

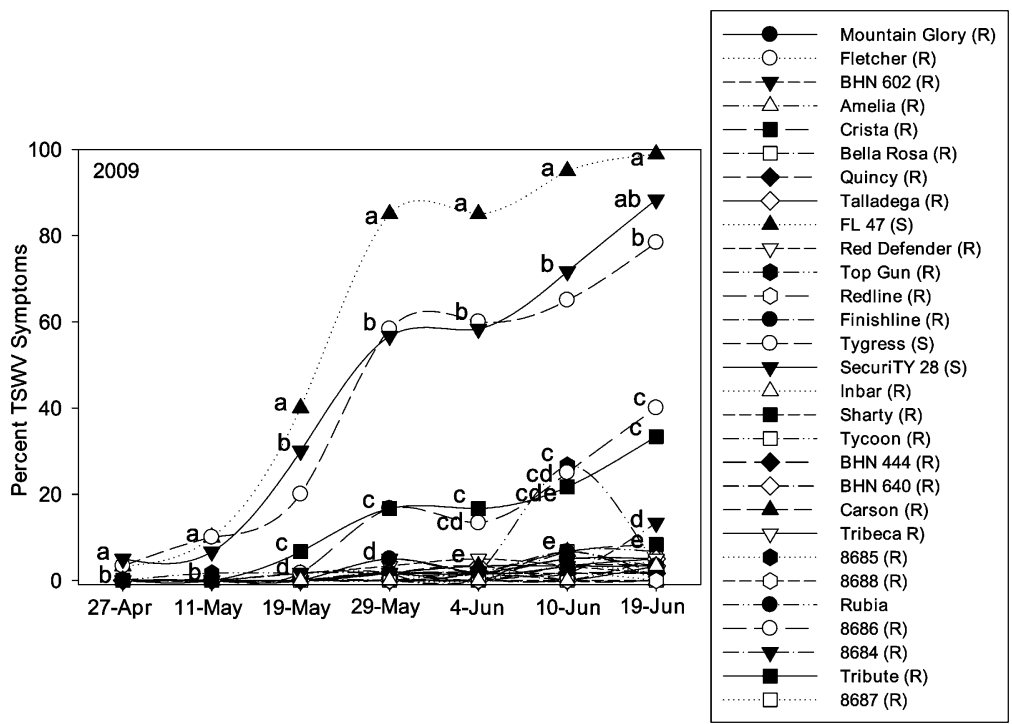

Fig. 5. The incidence of Tomato spotted wilt symptom in 2009. Means followed by the same letter within a sample date are not significantly different (least significant difference test, $P<0.05$ ). progression curves showed a high incidence of TSW symptoms on 'Caporal', 'FL 47', 'Marglobe', and 'Mariana', ranging from $25 \%$ to $98 \%$ between 18 May and 5 June 2006 (Fig. 1). The TSW incidence was confirmed with DAS ELISA and there was no significant difference in percent viruliferous plants per plot between cultivars using ELISApositive counts (range, $0 \%$ to $100 \%$; mean \pm $\left.\mathrm{SD}=21 \% \pm 34 \% ; F_{10,20}=1.9 ; P=0.093\right)$. The lack of symptom expression in TSWresistant cultivars translated into significantly greater marketable fruit yield than on the susceptible cultivars. Among several resistant cultivars tested in 2006, the most notable ones were 'Amelia', 'Quincy', 'Inbar', and 'Crista'. Unmarketable fruit weight and number were greatest for 'Caporal'. These fruits were mostly damaged by TSW symptoms, but other pest injury was noted as well. Total thrips densities found in the beat-cup samples were not significantly different among tomato cultivars (Table 2). The thrips specimens collected included males and females of $F$. fusca, $F$. occidentalis, and other species, but $F$. fusca females dominated this group.

Overall TSW disease incidence was lower in 2007 than in 2006 based on average TSW symptom expressed in susceptible cultivars (Table 3). On 18 May 2007, TSW disease symptom was significantly more apparent on 'FL 47' than on others cultivars (Fig. 3) and in the next week, all entries labeled susceptible had TSW symptoms. On 29 May, TSW symptoms were highest on 'Caporal'. The cultivars Crista, Talladega, Bella Rosa, Amelia, Redline, BHN 602, and Quincy produced significantly more marketable fruit weight than in TSW-susceptible lines. Also, the percent TSW fruit damage was significantly less $\left(F_{19,57}=3.7 ; P<0.001\right)$ on resistant cultivars (Fig. 2). With the exception of 'Cupid' (a grape sized tomato), the number of marketable tomatoes was not distinctly different among cultivars (Table 3 ). In addition, significantly higher unmarketable tomato fruits by weight and number occurred in the TSW susceptible cultivar Caporal.

Table 2. Marketable fruit yield per six plants and Tomato spotted wilt (TSW) fruit damage among tomato cultivars in 2006.

\begin{tabular}{|c|c|c|c|c|c|c|c|}
\hline \multirow[b]{2}{*}{ Cultivar $^{\mathrm{y}}$} & \multicolumn{3}{|c|}{ Marketable fruit yield } & \multicolumn{2}{|c|}{ Unmarketable fruit yield } & \multirow[b]{2}{*}{ TSW symptom ${ }^{\mathrm{w}}(\%)$} & \multirow[b]{2}{*}{ Incidence of thrips } \\
\hline & $\overline{\mathrm{Wt}}(\mathrm{kg})$ & No. of fruits & Price value $^{\mathrm{x}}(\$)$ & Wt (kg) & No. of fruits & & \\
\hline$\overline{\text { Amelia }(\mathrm{R})^{\mathrm{z}}}$ & $7.1 \mathrm{a}^{\mathrm{u}}$ & $39.7 \mathrm{~b}$ & 5.6 & $5.6 \mathrm{~b}-\mathrm{d}$ & $34.0 \mathrm{c}$ & $0.8 \mathrm{~d}$ & $19.3 \mathrm{a}$ \\
\hline Quincy (R) & $6.8 \mathrm{a}$ & $38.0 \mathrm{~b}$ & 5.4 & $3.1 \mathrm{~cd}$ & $16.7 \mathrm{c}$ & $0.8 \mathrm{~d}$ & $49.7 \mathrm{a}$ \\
\hline Inbar (R) & $6.5 \mathrm{a}$ & $37.0 \mathrm{~b}$ & 5.1 & $5.9 \mathrm{a}-\mathrm{c}$ & $32.7 \mathrm{c}$ & $5.0 \mathrm{~d}$ & $25.3 \mathrm{a}$ \\
\hline Crista (R) & $6.1 \mathrm{a}$ & $35.0 \mathrm{~b}$ & 4.9 & $3.6 \mathrm{~cd}$ & $18.0 \mathrm{c}$ & $7.5 \mathrm{~d}$ & $19.7 \mathrm{a}$ \\
\hline Bella Rosa (R) & $5.7 \mathrm{ab}$ & $39.0 \mathrm{~b}$ & 4.5 & $5.0 \mathrm{~b}-\mathrm{d}$ & $32.3 \mathrm{c}$ & $4.2 \mathrm{~d}$ & $19.7 \mathrm{a}$ \\
\hline BHN $640(\mathrm{R})$ & $4.7 \mathrm{ab}$ & $30.0 \mathrm{~b}$ & 3.7 & $7.4 \mathrm{ab}$ & $48.3 \mathrm{c}$ & $7.5 \mathrm{~d}$ & $16.3 \mathrm{a}$ \\
\hline Caporal (S) & $2.9 \mathrm{bc}$ & $40.0 \mathrm{~b}$ & 2.3 & $9.5 \mathrm{a}$ & $130.0 \mathrm{~b}$ & $52.5 \mathrm{c}$ & $15.0 \mathrm{a}$ \\
\hline FL $47(\mathrm{~S})$ & $1.5 \mathrm{c}$ & $7.3 \mathrm{~b}$ & 1.2 & $3.6 \mathrm{~cd}$ & $30.3 \mathrm{c}$ & $67.5 \mathrm{~b}$ & $19.3 \mathrm{a}$ \\
\hline Mariana (S) & $1.2 \mathrm{c}$ & $10.3 \mathrm{~b}$ & 0.9 & $2.9 \mathrm{~cd}$ & $44.0 \mathrm{c}$ & $40.5 \mathrm{a}$ & $37.7 \mathrm{a}$ \\
\hline Cupid (S) & $0.8 \mathrm{c}$ & $112.3 \mathrm{a}$ & 0.6 & $2.2 \mathrm{~d}$ & $254.0 \mathrm{a}$ & $5.0 \mathrm{~d}$ & $30.0 \mathrm{a}$ \\
\hline Marglobe (S) & $0.4 \mathrm{c}$ & $2.7 \mathrm{~b}$ & 0.3 & $4.2 \mathrm{~b}-\mathrm{d}$ & $43.0 \mathrm{c}$ & $83.3 \mathrm{a}$ & $15.0 \mathrm{a}$ \\
\hline $\mathrm{F}_{(\mathrm{df1} 1, \mathrm{df} 2)}$ & $6.2_{(10,20)}$ & $3.7_{(10,20)}$ & - & $3 \cdot 1_{(10,20)}$ & $11.0_{(10,20)}$ & $43.3_{(10,119)}$ & $1.3_{(10,20)}$ \\
\hline$P^{(\operatorname{ar1}, \mathrm{al} 2)}$ & $* * *$ & $* *$ & - & $*$ & *** & $* * *$ & NS \\
\hline
\end{tabular}

The marketable and unmarketable yield data represent samples collected on 6, 15, and 20 June 2006.

${ }^{2}$ Previously classified resistant or susceptible cultivars; $\mathrm{R}=$ resistant; $\mathrm{S}=$ susceptible.

${ }^{y}$ Cultivars sorted on marketable fruit wt (from heaviest to lightest).

${ }^{x}$ Price value set by USDA National Agricultural Statistical Service as $\$ 0.79 / \mathrm{kg}$ in Georgia.

${ }^{\text {w} M e a n ~ T S W ~ s y m p t o m s ~ r e c o r d e d ~ p e r ~ p l a n t ~ p e r ~ s e a s o n . ~}$

${ }^{ }$As per beat-cup samples collected on 2 May 2006.

"Means followed by the same letter within the column (cultivars) are not significantly different (least significant difference test, $P<0.05$ ).

NS, *, **, *** represent non-significant at $P<0.05$ or $P \leq 0.05,0.01$, and 0.001 , respectively. 
Table 3. Marketable fruit yield and Tomato spotted wilt (TSW) fruit damage per six plants among tomato cultivars in 2007.

\begin{tabular}{|c|c|c|c|c|c|c|}
\hline \multirow[b]{2}{*}{ Cultivarr $^{\mathrm{y}}$} & \multicolumn{3}{|c|}{ Marketable fruit yield } & \multicolumn{2}{|c|}{ Unmarketable fruit yield } & \multirow[b]{2}{*}{ TSW symptom ${ }^{\mathrm{w}}(\%)$} \\
\hline & Wt $(\mathrm{kg})$ & No. of fruits & Price value $^{\mathrm{x}}(\$)$ & Wt (kg) & No. of fruits & \\
\hline Talladega (R) & $26.7 \mathrm{ab}$ & $147.3 \mathrm{~b}-\mathrm{e}$ & 16.8 & $2.9 \mathrm{c}-\mathrm{f}$ & $20.5 \mathrm{~cd}$ & $0.0 \mathrm{c}$ \\
\hline Redline (R) & $23.5 \mathrm{a}-\mathrm{c}$ & $130.5 \mathrm{c}-\mathrm{e}$ & 14.8 & $2.2 \mathrm{ef}$ & $11.3 \mathrm{~d}$ & $0.5 \mathrm{c}$ \\
\hline BHN 602 (R) & $23.5 \mathrm{a}-\mathrm{c}$ & $138.3 \mathrm{~b}-\mathrm{e}$ & 14.8 & $2.9 \mathrm{c}-\mathrm{f}$ & $20.3 \mathrm{~cd}$ & $1.1 \mathrm{c}$ \\
\hline Quincy(R) & $23.3 \mathrm{a}-\mathrm{c}$ & $134.3 \mathrm{c}-\mathrm{e}$ & 14.7 & $2.5 \mathrm{ef}$ & $15.3 \mathrm{~cd}$ & $0.2 \mathrm{c}$ \\
\hline Red Defender(R) & $21.6 \mathrm{~b}-\mathrm{e}$ & $130.5 \mathrm{c}-\mathrm{e}$ & 13.6 & $2.1 \mathrm{ef}$ & $19.5 \mathrm{~cd}$ & $0.5 \mathrm{c}$ \\
\hline BHN $444(\mathrm{R})$ & $21.2 \mathrm{~b}-\mathrm{e}$ & $219.5 \mathrm{bc}$ & 13.3 & $2.2 \mathrm{ef}$ & $17.5 \mathrm{~cd}$ & $0.2 \mathrm{c}$ \\
\hline Picus (R) & $20.2 \mathrm{~b}-\mathrm{e}$ & $243.3 \mathrm{~b}$ & 12.7 & $1.6 \mathrm{f}$ & $27.0 \mathrm{~cd}$ & $0.2 \mathrm{c}$ \\
\hline BHN 640 (R) & $19.5 \mathrm{~b}-\mathrm{f}$ & $115.3 \mathrm{c}-\mathrm{e}$ & 12.3 & $2.7 \mathrm{~d}-\mathrm{f}$ & $20.3 \mathrm{~cd}$ & $0.5 \mathrm{c}$ \\
\hline $\operatorname{Inbar}(\mathrm{R})$ & $18.9 \mathrm{~b}-\mathrm{f}$ & $122.0 \mathrm{c}-\mathrm{e}$ & 11.9 & $2.6 \mathrm{~d}-\mathrm{f}$ & $16.3 \mathrm{~cd}$ & $2.9 \mathrm{bc}$ \\
\hline Muriel (R) & $17.7 \mathrm{c}-\mathrm{f}$ & $182.3 \mathrm{~b}-\mathrm{d}$ & 11.1 & $3.4 \mathrm{~b}-\mathrm{f}$ & $39.3 \mathrm{~cd}$ & $1.4 \mathrm{c}$ \\
\hline Caporal (S) & $15.1 \mathrm{~d}-\mathrm{g}$ & $164.3 \mathrm{~b}-\mathrm{e}$ & 9.5 & $8.3 \mathrm{a}$ & 107.3 b & $13.3 \mathrm{a}$ \\
\hline $\mathrm{F}_{(\mathrm{df1}, \mathrm{df2})}$ & $\begin{array}{l}\left.4.6_{(19}, 57\right) \\
* * *\end{array}$ & $20.7_{(19,57)}$ & - & $4.5_{(19,57)}$ & $\begin{array}{l}20.7_{(19,57)} \\
* * * *\end{array}$ & $9.1_{(19,537)}$ \\
\hline$P$ & *** & $* * *$ & - & $* * *$ & $* * *$ & $* * *$ \\
\hline
\end{tabular}

The marketable and unmarketable yield data represent samples collected on 20 and 28 June in 2007.

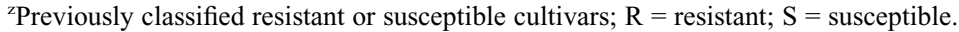

${ }^{y}$ Cultivars sorted on marketable fruit wt (from heaviest to lightest).

xPrice value set by USDA National Agricultural Statistical Service as $\$ 0.63 / \mathrm{kg}$ tomatoes in Georgia.

${ }^{\mathrm{w}}$ Mean TSW symptom recorded per plant per season.

${ }^{\mathrm{v}}$ Means followed by the same letter within the column within the crop are not significantly different (least significant difference test, $P<0.05$ ).

Ns, *** represent non-significant at $P<0.05$ or $P<0.001$, respectively.

Table 4. Evaluation of marketable fruit yield and Tomato spotted wilt (TSW) fruit damage per six plants among tomato cultivars in 2008.

\begin{tabular}{|c|c|c|c|c|c|c|c|}
\hline \multirow[b]{2}{*}{ Cultivar ${ }^{y}$} & \multicolumn{3}{|c|}{ Marketable fruit yield } & \multicolumn{2}{|c|}{ Unmarketable fruit yield } & \multirow[b]{2}{*}{ TSW symptom ${ }^{\mathrm{w}}(\%)$} & \multirow[b]{2}{*}{ Incidence of thrips } \\
\hline & Wt (kg) & No. of fruits & Price value ${ }^{\mathrm{x}}(\$)$ & $\overline{W t}(\mathrm{~kg})$ & No. of fruits & & \\
\hline Tous $91(\mathrm{R})$ & $20.3 \mathrm{ab}$ & $90.0 \mathrm{~b}-\mathrm{d}$ & 14.4 & $1.9 \mathrm{a}$ & $8.6 \mathrm{hi}$ & $0.9 \mathrm{~d}$ & $14.4 \mathrm{a}$ \\
\hline Quincy (R) & $19.6 \mathrm{a}-\mathrm{d}$ & $97.0 \mathrm{~b}-\mathrm{d}$ & 13.9 & $3.0 \mathrm{a}$ & $16.3 \mathrm{~g}-\mathrm{i}$ & $0.6 \mathrm{~d}$ & $13.3 \mathrm{a}$ \\
\hline Nico $(R)$ & $19.1 \mathrm{a}-\mathrm{d}$ & $103.0 \mathrm{~b}-\mathrm{d}$ & 13.4 & $1.5 \mathrm{a}$ & $8.6 \mathrm{hi}$ & $3.4 \mathrm{~cd}$ & $15.9 \mathrm{a}$ \\
\hline Talladega (R) & $18.4 \mathrm{a}-\mathrm{d}$ & $90.3 \mathrm{~b}-\mathrm{d}$ & 13.0 & $3.1 \mathrm{a}$ & $18.0 \mathrm{f}-\mathrm{i}$ & $1.9 \mathrm{~cd}$ & $13.7 \mathrm{a}$ \\
\hline Shanty (R) & $17.1 \mathrm{a}-\mathrm{d}$ & $140.0 \mathrm{~b}-\mathrm{d}$ & 12.1 & $3.8 \mathrm{a}$ & $28.0 \mathrm{c}-\mathrm{i}$ & $0.3 \mathrm{~d}$ & $13.8 \mathrm{a}$ \\
\hline FL $47(\mathrm{~S})$ & $17.1 \mathrm{a}-\mathrm{d}$ & $87.0 \mathrm{~b}-\mathrm{d}$ & 12.1 & $3.9 \mathrm{a}$ & $24.0 \mathrm{e}-\mathrm{i}$ & $10.4 \mathrm{ab}$ & $8.2 \mathrm{a}$ \\
\hline Top Gun (R) & $16.9 \mathrm{a}-\mathrm{d}$ & $85.7 \mathrm{~b}-\mathrm{d}$ & 11.9 & $3.2 \mathrm{a}$ & $21.7 \mathrm{f}-\mathrm{i}$ & $0.3 \mathrm{~d}$ & $13.9 \mathrm{a}$ \\
\hline BHN 444 (R) & $16.2 \mathrm{~b}-\mathrm{e}$ & $69.3 \mathrm{~cd}$ & 11.5 & $3.9 \mathrm{a}$ & $28.0 \mathrm{~d}-\mathrm{i}$ & $3.2 \mathrm{~cd}$ & $10.0 \mathrm{a}$ \\
\hline Bella Rosa (R) & $16.0 \mathrm{~b}-\mathrm{e}$ & $81.0 \mathrm{~b}-\mathrm{d}$ & 11.4 & $5.0 \mathrm{a}$ & $30.3 \mathrm{~b}-\mathrm{i}$ & $0.7 \mathrm{~d}$ & $13.2 \mathrm{a}$ \\
\hline Mariana (S) & $15.8 \mathrm{~b}-\mathrm{e}$ & $177.7 \mathrm{~b}$ & 11.2 & $1.7 \mathrm{a}$ & $21.3 \mathrm{f}-\mathrm{i}$ & $10.2 \mathrm{ab}$ & $9.3 \mathrm{a}$ \\
\hline BHN 602 (R) & $15.1 \mathrm{~b}-\mathrm{e}$ & $87.8 \mathrm{~b}-\mathrm{d}$ & 10.7 & $2.8 \mathrm{a}$ & $21.0 \mathrm{f}-\mathrm{i}$ & $0.6 \mathrm{~d}$ & $8.8 \mathrm{a}$ \\
\hline Muriel (R) & $12.9 \mathrm{~b}-\mathrm{e}$ & $116.0 \mathrm{~b}-\mathrm{d}$ & 9.1 & $5.3 \mathrm{a}$ & $52.7 \mathrm{ab}$ & $0.6 \mathrm{~d}$ & $11.9 \mathrm{a}$ \\
\hline Cupid (S) & $12.7 \mathrm{c}-\mathrm{e}$ & $945.8 \mathrm{a}$ & 9.0 & $0.8 \mathrm{a}$ & $50.3 \mathrm{a}-\mathrm{d}$ & $3.3 \mathrm{~cd}$ & $7.6 \mathrm{a}$ \\
\hline FTM 2305 (R) & $12.4 \mathrm{de}$ & $61.3 \mathrm{~d}$ & 8.8 & $1.5 \mathrm{a}$ & 8.7 hi & $1.5 \mathrm{~d}$ & $12.7 \mathrm{a}$ \\
\hline Caporal (S) & $9.4 \mathrm{e}$ & $110.3 \mathrm{~b}-\mathrm{d}$ & 6.6 & $3.7 \mathrm{a}$ & $41.3 \mathrm{a}-\mathrm{f}$ & $14.3 \mathrm{a}$ & $50.7 \mathrm{a}$ \\
\hline Pony Express (S) & $9.2 \mathrm{e}$ & $108.3 \mathrm{~b}-\mathrm{d}$ & 6.5 & $3.8 \mathrm{a}$ & $51.7 \mathrm{a}-\mathrm{c}$ & $14.9 \mathrm{a}$ & $8.8 \mathrm{a}$ \\
\hline Hedvig (R) & $9.1 \mathrm{e}$ & $97.0 \mathrm{~b}-\mathrm{d}$ & 6.4 & $4.4 \mathrm{a}$ & $55.3 \mathrm{a}$ & $1.5 \mathrm{~d}$ & $7.9 \mathrm{a}$ \\
\hline Galilea (R) & $8.8 \mathrm{e}$ & $76.3 \mathrm{~cd}$ & 6.4 & $3.8 \mathrm{a}$ & $40.3 \mathrm{a}-\mathrm{g}$ & $4.1 \mathrm{~cd}$ & $12.9 \mathrm{a}$ \\
\hline $\mathrm{F}_{(\mathrm{df1} 1, \mathrm{df} 2)}$ & $2.1_{(27,54)}$ & $22.1_{(27,54)}$ & - & $1.5_{(27,54)}$ & $3.3_{(27,54)}$ & $4.2_{(27,559)}$ & $1.7_{(27,54)}$ \\
\hline$P$ & $* *$ & $* * *$ & - & NS & $* * *$ & $* * *$ & NS \\
\hline
\end{tabular}

The marketable and unmarketable yield data represent samples collected on 25 June and 8 July in 2008.

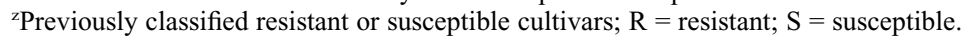

${ }^{\mathrm{y}}$ Cultivars sorted on marketable fruit wt (from heaviest to lightest).

${ }^{x}$ Price value set by USDA National Agricultural Statistical Service as $\$ 0.71 / \mathrm{kg}$ tomato in Georgia.

${ }^{\text {w} M e a n ~ T S W ~ s y m p t o m ~ r e c o r d e d ~ p e r ~ p l a n t ~ p e r ~ s e a s o n . ~}$

${ }^{v}$ As per beat-cup samples collected on 23 Apr. and 7 May and blossom samples on 5 June 2008.

"Means followed by the same letter within the column within the crop are not significantly different (least significant difference test, $P<0.05$ ).

Ns, *** represent non-significant at $P<0.05$ or $P \leq 0.001$, respectively. 
Table 5. Evaluation of marketable fruit yield and Tomato spotted wilt (TSW) fruit damage per six plants among tomato cultivars in 2009.

\begin{tabular}{|c|c|c|c|c|c|c|c|}
\hline \multirow[b]{2}{*}{ Cultivar $^{y}$} & \multicolumn{3}{|c|}{ Marketable fruit yield } & \multicolumn{2}{|c|}{ Unmarketable fruit yield } & \multirow{2}{*}{$\begin{array}{c}\text { TSW } \\
\text { symptom }^{w} \\
(\%)\end{array}$} & \multirow{2}{*}{$\begin{array}{c}\text { Incidence } \\
\text { of } \\
\text { thrips }^{\mathrm{v}}\end{array}$} \\
\hline & $\begin{array}{l}\text { Wt } \\
(\mathrm{kg})\end{array}$ & $\begin{array}{l}\text { No. of } \\
\text { fruits }\end{array}$ & $\begin{array}{c}\text { Price } \\
\text { value }^{\mathrm{x}}(\$)\end{array}$ & $\begin{array}{c}\mathrm{Wt} \\
(\mathrm{kg})\end{array}$ & $\begin{array}{c}\text { No. of } \\
\text { fruits }\end{array}$ & & \\
\hline Tycoon $(\mathrm{R})^{\mathrm{z}}$ & $36.1 \mathrm{a}^{\mathrm{u}}$ & $174.5 \mathrm{bc}$ & 39.3 & $9.6 \mathrm{a}$ & $37.5 \mathrm{~b}-\mathrm{f}$ & $0.5 \mathrm{~d}$ & $4.7 \mathrm{a}$ \\
\hline BHN 444 (R) & $30.1 \mathrm{ab}$ & $151.3 \mathrm{c}-\mathrm{f}$ & 32.8 & $7.6 \mathrm{a}-\mathrm{c}$ & $38.0 \mathrm{~b}-\mathrm{f}$ & $0.5 \mathrm{~d}$ & $5.0 \mathrm{a}$ \\
\hline Tribute & $27.6 \mathrm{bc}$ & $168.3 b-d$ & 30.0 & $5.8 \mathrm{~b}-\mathrm{f}$ & $30.0 \mathrm{e}-\mathrm{g}$ & $0.5 \mathrm{~d}$ & $6.5 \mathrm{a}$ \\
\hline BHN $640(\mathrm{R})$ & $27.1 \mathrm{bcd}$ & $159.5 \mathrm{c}-\mathrm{e}$ & 29.5 & $6.8 \mathrm{~b}-\mathrm{d}$ & $37.8 \mathrm{~b}-\mathrm{f}$ & $0.7 \mathrm{~d}$ & $6.5 \mathrm{a}$ \\
\hline $\operatorname{Inbar}(\mathrm{R})$ & $26.9 \mathrm{bcd}$ & $148.3 \mathrm{c}-\mathrm{g}$ & 29.4 & $6.7 \mathrm{~b}-\mathrm{e}$ & $36.0 \mathrm{~b}-\mathrm{g}$ & $0.7 \mathrm{~d}$ & $6.4 \mathrm{a}$ \\
\hline Shanty (R) & $26.6 \mathrm{bcd}$ & $204.0 \mathrm{~b}$ & 29.0 & $5.7 \mathrm{c}-\mathrm{f}$ & $47.8 \mathrm{ab}$ & $0.0 \mathrm{~d}$ & $5.8 \mathrm{a}$ \\
\hline Rubia (R) & 25.4 bcde & $262.8 \mathrm{a}$ & 27.7 & $2.9 \mathrm{~g}$ & $34.3 \mathrm{~b}-\mathrm{g}$ & $2.1 \mathrm{~d}$ & $5.6 \mathrm{a}$ \\
\hline Amelia (R) & 5.1 bcde & $140.3 \mathrm{c}-\mathrm{h}$ & 27.3 & $6.7 \mathrm{~b}-\mathrm{e}$ & $36.3 \mathrm{~b}-\mathrm{g}$ & $2.1 \mathrm{~d}$ & $4.2 \mathrm{a}$ \\
\hline $\begin{array}{l}\text { Mountain } \\
\text { Glory (R) }\end{array}$ & $24.6 \mathrm{bcdef}$ & $123.8 \mathrm{e}-\mathrm{i}$ & 26.8 & $6.2 \mathrm{~b}-\mathrm{e}$ & $30.0 \mathrm{e}-\mathrm{g}$ & $1.9 \mathrm{~d}$ & $6.4 \mathrm{a}$ \\
\hline Fletcher (R) & 24.5 bcdef & $148.0 \mathrm{c}-\mathrm{g}$ & 26.6 & $6.5 \mathrm{~b}-\mathrm{f}$ & $30.7 \mathrm{~d}-\mathrm{g}$ & $0.5 \mathrm{~d}$ & $5.4 \mathrm{a}$ \\
\hline Bella Rosa (R) & 24.4 bcdef & $121.8 \mathrm{f}-\mathrm{i}$ & 26.6 & $6.9 \mathrm{a}-\mathrm{d}$ & $35.0 \mathrm{~b}-\mathrm{g}$ & $0.9 \mathrm{~d}$ & $5.5 \mathrm{a}$ \\
\hline $\begin{array}{l}\text { Red } \\
\quad \text { Defender (R) }\end{array}$ & 23.6 cdefg & $140.8 \mathrm{c}-\mathrm{h}$ & 25.2 & $5.4 \mathrm{c}-\mathrm{g}$ & $30.0 \mathrm{e}-\mathrm{g}$ & $1.7 \mathrm{~d}$ & $4.4 \mathrm{a}$ \\
\hline Carson (R) & 22.9 cdefg & $134.5 \mathrm{e}-\mathrm{i}$ & 24.9 & $6.8 \mathrm{~b}-\mathrm{d}$ & $37.5 \mathrm{~b}-\mathrm{f}$ & $0.7 \mathrm{~d}$ & $3.5 \mathrm{a}$ \\
\hline $8688(\mathrm{R})$ & $.8 \mathrm{c}-\mathrm{g}$ & $134.8 \mathrm{e}-\mathrm{i}$ & 24.8 & $5.4 \mathrm{c}-\mathrm{g}$ & $30.3 \mathrm{e}-\mathrm{g}$ & $0.0 \mathrm{~d}$ & $4.4 \mathrm{a}$ \\
\hline Tribeca (R) & $.5 \mathrm{c}-\mathrm{g}$ & $123.8 \mathrm{e}-\mathrm{i}$ & 24.6 & $6.4 \mathrm{~b}-\mathrm{f}$ & $33.0 \mathrm{c}-\mathrm{g}$ & $2.1 \mathrm{~d}$ & $3.9 \mathrm{a}$ \\
\hline & $2.4 \mathrm{c}-\mathrm{g}$ & $122.5 \mathrm{e}-\mathrm{i}$ & 24.4 & $6.1 \mathrm{~b}-\mathrm{f}$ & $29.0 \mathrm{e}-\mathrm{g}$ & $0.7 \mathrm{~d}$ & $4.5 \mathrm{a}$ \\
\hline Quincy (R) & $22.2 \mathrm{c}-\mathrm{g}$ & $168.0 \mathrm{e}-\mathrm{i}$ & 23.9 & $6.9 \mathrm{a}-\mathrm{d}$ & $35.5 \mathrm{~b}-\mathrm{g}$ & $0.9 \mathrm{~d}$ & $5.7 \mathrm{a}$ \\
\hline Crista & $21.6 \mathrm{c}-\mathrm{g}$ & $121.8 \mathrm{f}-\mathrm{i}$ & 23.5 & $3.9 \mathrm{e}-\mathrm{g}$ & $23.5 \mathrm{~g}$ & $2.4 \mathrm{~d}$ & $5.1 \mathrm{a}$ \\
\hline BHN 602 (R) & $1.5 \mathrm{c}-\mathrm{g}$ & $125.5 \mathrm{e}-\mathrm{i}$ & 23.4 & $7.2 \mathrm{a}-\mathrm{d}$ & $36.0 \mathrm{~b}-\mathrm{g}$ & $0.9 \mathrm{~d}$ & $6.5 \mathrm{a}$ \\
\hline Talladega (R) & $.5 \mathrm{~d}-\mathrm{h}$ & $109.0 \mathrm{e}-\mathrm{i}$ & 22.2 & $6.4 \mathrm{~b}-\mathrm{f}$ & $30.5 \mathrm{~d}-\mathrm{g}$ & $2.1 \mathrm{~d}$ & $4.5 \mathrm{a}$ \\
\hline Finishline $(\mathrm{R})$ & $19.4 \mathrm{e}-\mathrm{h}$ & $104.5 \mathrm{hi}$ & 21.1 & $8.4 \mathrm{ab}$ & $44.3 \mathrm{a}-\mathrm{d}$ & $1.9 \mathrm{~d}$ & $6.1 \mathrm{a}$ \\
\hline Redline & $9.1 \mathrm{e}-\mathrm{h}$ & $107.5 \mathrm{hi}$ & 20.8 & $7.5 \mathrm{a}-\mathrm{c}$ & $39.0 \mathrm{a}-\mathrm{e}$ & $0.5 \mathrm{~d}$ & $6.7 \mathrm{a}$ \\
\hline Top Gun (R) & $18.1 \mathrm{f}-\mathrm{h}$ & $111.5 \mathrm{~g}-\mathrm{i}$ & 19.8 & $3.7 \mathrm{f}-\mathrm{h}$ & $24.5 \mathrm{fg}$ & $5.2 \mathrm{~d}$ & $3.3 \mathrm{a}$ \\
\hline $8684(\mathrm{R})$ & $18.1 \mathrm{f}-\mathrm{i}$ & $99.8 \mathrm{i}$ & 19.7 & $5.2 \mathrm{c}-\mathrm{g}$ & $30.7 \mathrm{~d}-\mathrm{g}$ & $2.9 \mathrm{~d}$ & $3.7 \mathrm{a}$ \\
\hline $8687(\mathrm{R})$ & $17.5 \mathrm{gh}$ & $98.8 \mathrm{i}$ & 19.0 & $6.1 \mathrm{~b}-\mathrm{f}$ & $33.7 \mathrm{c}-\mathrm{g}$ & $13.6 \mathrm{c}$ & $4.7 \mathrm{a}$ \\
\hline 8686 (R) & $14.3 \mathrm{~h}$ & $109.5 \mathrm{hi}$ & 15.6 & $7.3 \mathrm{a}-\mathrm{d}$ & $52.3 \mathrm{a}$ & $13.8 \mathrm{c}$ & $3.9 \mathrm{a}$ \\
\hline Tygress (S) & $6.7 \mathrm{i}$ & $45.5 \mathrm{j}$ & 7.2 & $4.5 \mathrm{~d}-\mathrm{g}$ & $34.5 \mathrm{~b}-\mathrm{g}$ & $42.1 \mathrm{~b}$ & $6.7 \mathrm{a}$ \\
\hline $\begin{array}{c}\text { SecuriTY } \\
28(\mathrm{~S})\end{array}$ & $5.2 \mathrm{i}$ & $30.3 \mathrm{j}$ & 5.6 & $7.0 \mathrm{a}-\mathrm{d}$ & $45.0 \mathrm{a}-\mathrm{c}$ & $45.2 \mathrm{~b}$ & $8.3 \mathrm{a}$ \\
\hline FL 47 (S) & $3.0 \mathrm{i}$ & $23.0 \mathrm{j}$ & 3.3 & $2.9 \mathrm{~g}$ & $29.3 \mathrm{e}-\mathrm{g}$ & $59.5 \mathrm{a}$ & $3.9 \mathrm{a}$ \\
\hline $\begin{array}{l}\mathrm{F}_{(\mathrm{df} 1, \mathrm{df} 2)} \\
P\end{array}$ & $\begin{array}{l}8.5_{(28,84)} \\
* * *\end{array}$ & $\begin{array}{l}12.8_{(28,84)} \\
\quad * * *\end{array}$ & - & $\begin{array}{c}2.4_{(28,84)} \\
* *\end{array}$ & $\begin{array}{c}1.7_{(28,84)} \\
\quad *\end{array}$ & $\begin{array}{c}36.9_{(28,780)} \\
* * *\end{array}$ & $\begin{array}{c}1.2_{(28,84)} \\
\text { NS }\end{array}$ \\
\hline
\end{tabular}

The marketable and unmarketable yield data represent samples collected on 17 and 29 June and 13 July in 2009.

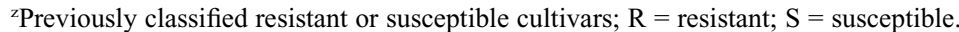

${ }^{\mathrm{y}}$ Cultivars sorted on marketable fruit wt (from heaviest to lightest).

xPrice value set by USDA National Agricultural Statistical Service as $\$ 1.08 / \mathrm{kg}$ tomatoes in Georgia.

${ }^{\text {w} M e a n ~ T S W ~ s y m p t o m ~ r e c o r d e d ~ p e r ~ p l a n t ~ p e r ~ s e a s o n . ~}$

${ }^{\mathrm{v} A s}$ per beat-cup samples collected on 29 Apr., 7 and 15 May, and blossom samples 22 and 29 May in 2009.

"Means followed by the same letter within the column within the crop are not significantly different (least significant difference test, $P<0.05$ ).

Ns, **, *** represent non-significant at $P<0.05$ or $P \leq 0.01$, and 0.001 , respectively.

In 2008, susceptible cultivars averaged $10 \%$ to $15 \%$ TSW-symptomatic plants (Table 4). On 28 May, 'Pony Express', 'Caporal', and 'FL 47' had significantly higher TSW symptoms $(\approx 10 \%$ to $20 \%)$ relative to other cultivars (Fig. 4). By 2 June, disease severely significantly intensified on 'Pony Express' and 'Caporal' ( $\approx 28 \%$ to $35 \%$ ) followed by 'Mariana', 'SecuriTY 28', 'Tygress', and 'FL 47' compared with resistant cultivars. The overall disease progress was similar to 2007 (Fig. 3) but slightly delayed compared with 2006 (Fig. 1) and 2009 (Fig. 5). Also, marketable fruit weight was not distinctly different among cultivars tested as a result of the relatively low incidence of TSW. Total thrips in both beat-cup and blossom samples combined did not show any significant difference among tomato cultivars (Table 4 ).

Compared with previous years, average TSW incidence was highest in 2009, averaging $60 \%$ over the season in 'FL 47 ' (Table 5) and reaching an average of $99 \%$ at the time of harvest. On average, TSW symptom was the most severe on 'FL 47' followed by 'Tygress' and 'SecuriTY 28', than on experimental hybrids 8686 and 8687 and least on the other resistant cultivars. On initial scouting (27 Apr. 2009), TSW disease symptoms were detected on the susceptible cultivars, especially FL 47, Tygress, and SecuriTY 28. These symptoms intensified to $70 \%$ to $98 \%$ on the last scouting (Fig. 5). Between 29 May and 19 June, TSW symptoms remained high in susceptible cultivars. Comparing Figures 3, 4, and 5, the high incidence of TSW in 2009 occurred because of the higher early-season TSW pressure compared with the previous 2 years.

Symptoms were significantly more apparent on two of the resistant experimental hybrids 8686 and 8687 compared with other resistance lines. Because resistance was intermediate, we expect some contamination had occurred in the seed stock. Even so, all resistant cultivars provided significantly higher marketable yield (Table 5). The top 10 culti- vars based of marketable weight under this severe TSW pressure were: 'Tycoon', 'BHN 444', 'Tribute', 'BHN 640', 'Inbar', 'Shanty' (Roma type), 'Rubia' (Roma type), 'Amelia', and 'Tribeca' and 'Mountain Glory' (Table 5). Results showed that the Roma hybrid 'Rubia' had the most number of fruits followed by 'Shanty', 'Tycoon', 'Tribute', and 'BHN 640'. Unmarketable fruit weight was greatest for 'Tycoon' and least for 'FL 47'. Because yield was so poor for the susceptible cultivars, the ranking of damaged fruit only seemed to suggest that higher-yielding commercial cultivars also experienced proportionally greater numbers of culled fruit. Overall density of thrips sampled from beat-cup and blossom did not differ among cultivars (Table 5).

In 2010, TSW pressure on tomato cultivars was the lowest compared with previous years. Even so, the greatest TSW symptom was observed on 'FL 47' $(\approx 5 \%)$ followed by 'Big Boy', 'SecuriTY 28', and 'Tygress' (Table 6). A few symptoms appeared by 16 Apr. 2010 on susceptible lines but did not increase during the rest of the season. No significant differences were detected among cultivars based on fruit weight; however, number of fruits varied among cultivars. 'Quincy', experimental hybrid '8749', 'Red Defender', 'Carson', and an experimental hybrid, '8746', had the most number of fruits. In addition, unmarketable fruits did not differ among cultivars by weight or number in the 2010 season. The low average TSW incidence was confirmed with DAS ELISA and there was no significant difference in percent viruliferous plants per plot between cultivars (range, $0 \%$ to $20 \%$; mean $\pm \mathrm{SD}=1 \% \pm 4 \% ; F_{29,87}=0.6 ; P=0.9$ ). Disease symptoms did not appear on any previously classified resistant cultivars. The beat-cup samples did not show any difference in thrips density $\left(F_{29,87}=0.9 ; P=0.542\right.$; Table 6).

Correlations of mean thrips with percent TSW symptomatic plants over all cultivars revealed no significant relationship with total thrips $\left(R_{350}=0.00, P=0.99\right)$ or $F$. fusca $\left(R_{350}=\right.$ $0.05, P=0.31)$ but a slight positive relationship with $F$. occidentalis $\left(R_{350}=0.18, P=\right.$ 0.001 ). On the other hand, only $F$. fusca had a negative relationship to marketable weight of tomatoes $\left(R_{353}=-0.33, P=0.0001\right)$. Thrips vectors were not consistently correlated with percent TSW in susceptible cultivars (e.g., only in 'FL 47' for the vector $F$. occidentalis, $R_{14}=0.60, P=0.02$ and in 'SecuriTY 28' for $F$. occidentalis and $F$. fusca, $R_{11}=0.87, P=$ $0.001, R_{11}=0.75, P=0.01$, respectively). In the resistant cultivars, significant correlations with $F$. fusca were detected in 'BHN 640', 'Bella Rosa', 'Top Gun', and 'Carson' $(P<$ $0.05)$, but the incidence of TSW averaged less than $1 \%$ in these cultivars. To summarize the thrips observations, we did not observe a trend of resistant cultivars having significantly fewer thrips nor did we see a consistent relationship between TSW and thrips within groups of resistant or susceptible tomato cultivars. Thus, TSW resistance in commercial tomato does not appear to be related to resistance to the thrips vectors. 
Table 6. Evaluation of marketable fruit yield and Tomato spotted wilt (TSW)fruit damage per six plants among tomato cultivars in 2010.

\begin{tabular}{|c|c|c|c|c|c|c|c|}
\hline \multirow[b]{2}{*}{ Cultivar $^{\mathrm{y}}$} & \multicolumn{3}{|c|}{ Marketable fruit yield } & \multicolumn{2}{|c|}{ Unmarketable fruit yield } & \multirow{2}{*}{$\begin{array}{c}\text { TSW } \\
\text { symptom }^{\mathrm{w}} \\
(\%)\end{array}$} & \multirow{2}{*}{$\begin{array}{c}\text { Incidence } \\
\text { of } \\
\text { thrips }^{\mathrm{v}}\end{array}$} \\
\hline & $\begin{array}{l}\mathrm{Wt} \\
(\mathrm{kg})\end{array}$ & $\begin{array}{l}\text { No. of } \\
\text { fruits }\end{array}$ & $\begin{array}{c}\text { Price } \\
\text { value }^{\mathrm{x}}(\$)\end{array}$ & $\begin{array}{c}\mathrm{Wt} \\
(\mathrm{kg})\end{array}$ & $\begin{array}{l}\text { No. of } \\
\text { fruits }\end{array}$ & & \\
\hline $8746(\mathrm{R})^{\mathrm{z}}$ & $26.4 \mathrm{a}^{\mathrm{u}}$ & $114.75 \mathrm{a}-\mathrm{d}$ & 28.12 & $2.9 \mathrm{a}$ & $15.0 \mathrm{a}$ & $0.0 \mathrm{e}$ & $6.5 \mathrm{a}$ \\
\hline Quincy (R) & $25.6 \mathrm{a}$ & $131.3 \mathrm{a}$ & 27.3 & $2.9 \mathrm{a}$ & $13.3 \mathrm{a}$ & $0.0 \mathrm{e}$ & $12.1 \mathrm{a}$ \\
\hline $\begin{array}{l}\text { Red } \\
\quad \text { Defender }(\mathrm{R})\end{array}$ & $23.6 \mathrm{a}$ & $116.0 \mathrm{a}-\mathrm{c}$ & 25.1 & $2.9 \mathrm{a}$ & $16.0 \mathrm{a}$ & $0.0 \mathrm{e}$ & $13.4 \mathrm{a}$ \\
\hline BHN $640(\mathrm{R})$ & $23.2 \mathrm{a}$ & $99.0 \mathrm{a}-\mathrm{g}$ & 24.7 & $2.9 \mathrm{a}$ & $15.0 \mathrm{a}$ & $0.0 \mathrm{e}$ & $4.4 \mathrm{a}$ \\
\hline Carson (R) & $22.9 \mathrm{a}$ & $117.3 \mathrm{a}-\mathrm{c}$ & 24.5 & $1.9 \mathrm{a}$ & $9.8 \mathrm{a}$ & $0.0 \mathrm{e}$ & $8.1 \mathrm{a}$ \\
\hline Talladega (R) & $21.5 \mathrm{a}$ & $103.3 \mathrm{a}-\mathrm{f}$ & 22.87 & $2.6 \mathrm{a}$ & $12.8 \mathrm{a}$ & $0.0 \mathrm{e}$ & $6.3 \mathrm{a}$ \\
\hline Tycoon (R) & $19.9 \mathrm{a}$ & $82.3 \mathrm{~b}-\mathrm{h}$ & 21.2 & $2.0 \mathrm{a}$ & $9.0 \mathrm{a}$ & $0.0 \mathrm{e}$ & $5.4 \mathrm{a}$ \\
\hline Top Gun (R) & $19.8 \mathrm{a}$ & $103.8 \mathrm{a}-\mathrm{f}$ & 21.1 & $0.8 \mathrm{a}$ & $5.0 \mathrm{a}$ & $0.0 \mathrm{e}$ & $4.4 \mathrm{a}$ \\
\hline Tribeca (R) & $19.7 \mathrm{a}$ & $102.5 \mathrm{a}-\mathrm{f}$ & 20.9 & $2.6 \mathrm{a}$ & $15.5 \mathrm{a}$ & $0.0 \mathrm{e}$ & $4.8 \mathrm{a}$ \\
\hline BHN 444 (R) & $19.4 \mathrm{a}$ & $98.5 \mathrm{a}-\mathrm{g}$ & 20.7 & $2.7 \mathrm{a}$ & $13.8 \mathrm{a}$ & $0.0 \mathrm{e}$ & $10.9 \mathrm{a}$ \\
\hline $8751(\mathrm{R})$ & $19.4 \mathrm{a}$ & $110.5 \mathrm{a}-\mathrm{e}$ & 20.7 & $2.4 \mathrm{a}$ & $12.3 \mathrm{a}$ & $0.0 \mathrm{e}$ & $7.3 \mathrm{a}$ \\
\hline Crista (R) & $19.1 \mathrm{a}$ & $101.0 \mathrm{a}-\mathrm{g}$ & 20.4 & $2.5 \mathrm{a}$ & $12.0 \mathrm{a}$ & $0.0 \mathrm{de}$ & $10.3 \mathrm{a}$ \\
\hline $8612(\mathrm{R})$ & $18.8 \mathrm{a}$ & $93.0 \mathrm{~b}-\mathrm{f}$ & 20.1 & $2.9 \mathrm{a}$ & $14.5 \mathrm{a}$ & $0.0 \mathrm{e}$ & $6.3 \mathrm{a}$ \\
\hline Redline (R) & $18.6 \mathrm{a}$ & $95.3 \mathrm{a}-\mathrm{f}$ & 19.8 & $3.1 \mathrm{a}$ & $16.3 \mathrm{a}$ & $0.0 \mathrm{e}$ & $5.1 \mathrm{a}$ \\
\hline 8749 (R) & $18.4 \mathrm{a}$ & $118.5 \mathrm{ab}$ & 19.6 & $2.1 \mathrm{a}$ & $12.5 \mathrm{a}$ & $0.0 \mathrm{e}$ & $5.8 \mathrm{a}$ \\
\hline BHN 602 (R) & $18.3 \mathrm{a}$ & $95.8 \mathrm{a}-\mathrm{g}$ & 19.5 & $1.4 \mathrm{a}$ & $8.5 \mathrm{a}$ & $0.0 \mathrm{e}$ & $5.6 \mathrm{a}$ \\
\hline Finishline (R) & $17.8 \mathrm{a}$ & $82.3 \mathrm{~b}-\mathrm{h}$ & 18.9 & $2.9 \mathrm{a}$ & $14.3 \mathrm{a}$ & $0.0 \mathrm{e}$ & $3.5 \mathrm{a}$ \\
\hline HAZ 3089 (R) & $17.7 \mathrm{a}$ & $70.75 \mathrm{f}-\mathrm{h}$ & 18.8 & $3.8 \mathrm{a}$ & $15.5 \mathrm{a}$ & $0.0 \mathrm{e}$ & $6.1 \mathrm{a}$ \\
\hline Tygress (S) & $17.6 \mathrm{a}$ & $81.3 \mathrm{~b}-\mathrm{h}$ & 18.8 & $1.2 \mathrm{a}$ & $6.0 \mathrm{a}$ & $1.3 \mathrm{~cd}$ & $9.9 \mathrm{a}$ \\
\hline $\begin{array}{c}\text { SecuriTY } \\
28(\mathrm{~S})\end{array}$ & $17.6 \mathrm{a}$ & $83.0 \mathrm{~b}-\mathrm{h}$ & 18.7 & $2.2 \mathrm{a}$ & $10.3 \mathrm{a}$ & $3.3 \mathrm{bc}$ & $8.1 \mathrm{a}$ \\
\hline $\begin{array}{l}\text { Mountain } \\
\text { Glory (R) }\end{array}$ & $17.4 \mathrm{a}$ & $85.3 \mathrm{~b}-\mathrm{h}$ & 18.6 & $1.6 \mathrm{a}$ & $8.5 \mathrm{a}$ & $0.0 \mathrm{e}$ & $9.1 \mathrm{a}$ \\
\hline HAZ 3084 (R) & $17.2 \mathrm{a}$ & $76.0 \mathrm{e}-\mathrm{h}$ & 18.3 & $2.1 \mathrm{a}$ & $8.3 \mathrm{a}$ & $0.0 \mathrm{e}$ & $5.6 \mathrm{a}$ \\
\hline $8768(\mathrm{R})$ & $16.8 \mathrm{a}$ & $80.5 \mathrm{c}-\mathrm{h}$ & 17.9 & $3.7 \mathrm{a}$ & $18.0 \mathrm{a}$ & $0.0 \mathrm{e}$ & $7.4 \mathrm{a}$ \\
\hline FL $47(\mathrm{~S})$ & $16.0 \mathrm{a}$ & $87.3 \mathrm{~b}-\mathrm{h}$ & 17.0 & $0.9 \mathrm{a}$ & $5.8 \mathrm{a}$ & $4.7 \mathrm{a}$ & $5.5 \mathrm{a}$ \\
\hline Fletcher (R) & $15.8 \mathrm{a}$ & $79.5 \mathrm{c}-\mathrm{h}$ & 16.9 & $2.5 \mathrm{a}$ & $14.0 \mathrm{a}$ & $0.0 \mathrm{e}$ & $6.3 \mathrm{a}$ \\
\hline $8793(\mathrm{R})$ & $14.9 \mathrm{a}$ & $77.0 \mathrm{~d}-\mathrm{h}$ & 15.9 & $3.1 \mathrm{a}$ & $14.8 \mathrm{a}$ & $0.0 \mathrm{e}$ & $4.3 \mathrm{a}$ \\
\hline Bella Rosa (R) & $14.6 \mathrm{a}$ & $69.0 \mathrm{f}-\mathrm{h}$ & 15.5 & $1.5 \mathrm{a}$ & $7.3 \mathrm{a}$ & $0.0 \mathrm{e}$ & $13.8 \mathrm{a}$ \\
\hline Amelia (R) & $14.1 \mathrm{a}$ & $64.3 \mathrm{gh}$ & 14.9 & $1.8 \mathrm{a}$ & $8.8 \mathrm{a}$ & $0.0 \mathrm{e}$ & $5.0 \mathrm{a}$ \\
\hline HAZ 3088 (R) & $12.5 \mathrm{a}$ & $54.3 \mathrm{~h}$ & 13.3 & $2.9 \mathrm{a}$ & $11.5 \mathrm{a}$ & $0.0 \mathrm{e}$ & $7.5 \mathrm{a}$ \\
\hline $\operatorname{Big} \operatorname{Boy}(\mathrm{S})$ & $12.1 \mathrm{a}$ & $81.0 \mathrm{~b}-\mathrm{h}$ & 12.9 & $2.3 \mathrm{a}$ & $13.3 \mathrm{a}$ & $3.1 \mathrm{~b}$ & $4.3 \mathrm{a}$ \\
\hline $\begin{array}{l}\mathrm{F}_{(\mathrm{df} 1, \mathrm{df} 2)} \\
P\end{array}$ & $\begin{array}{c}1.2_{(29,87)} \\
(-5 S\end{array}$ & $\begin{array}{c}1.7_{(29,87)} \\
*\end{array}$ & - & $0.8_{(29,87)}$ & $0.8_{(29,87)}$ & $\begin{array}{c}13.9_{(29,927)} \\
* * *\end{array}$ & $0.9_{(29,87)}$ \\
\hline
\end{tabular}

The marketable and unmarketable yield data represent samples collected on 8 and 22 June in 2010.

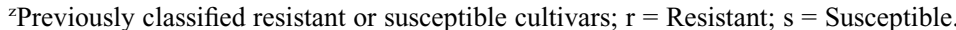

${ }^{\mathrm{y}}$ Cultivars sorted on marketable fruit wt (from heaviest to lightest).

xPrice value set by USDA National Agricultural Statistical Service as $\$ 1.1 / \mathrm{kg}$ tomato fruit on nation-wide survey in May 2010.

${ }^{w}$ Mean TSW symptom recorded per plant per season.

${ }^{v}$ As per beat-cup samples collected on 21 Apr. and 13 May 2010.

"Means followed by the same letter within the column within the crop are not significantly different (least significant difference test, $P<0.05$ ).

NS, **, *** represent non-significant at $P<0.05$ or $P \leq 0.01$, and 0.001 , respectively.

In this study, TSW disease pressure was severe enough to cause significant yield loss in 4 of 5 years. Under these conditions, the top 15 commercial cultivars based on consistent TSW resistance and ranked from highest marketable fruit yield were: 'Tycoon', 'Tous 91', 'Talladega', 'Red Defender', 'BHN 444', 'Nico', 'Carson', 'BHN 685 (Roma type)', 'Picus', 'Redline', 'Tribute', 'Quincy', 'BHN 640', 'BHN 602', and 'Top Gun' (Table 1). Previous research on $S w$-5-based resistance in 'BHN 444' demonstrated no TSW symptom expression in tomato foliage when exposed to both mild (GATb-1) and severe (GAL) TSWV isolates in Georgia (Mandal et al., 2006). Hybrids heterozygous for $\mathrm{Sw}-7$ had an intermediate amount of TSW incidence (Table 5). This was not expected based on previous observations of 39 heterozygous $F_{3}$ lines that fit the expected 3:1 ratio for control by a single dominant gene (Scott and Olson, unpublished data). The two hybrids, 8686 and 8687 , have the same $S w-7$ parent and there is a chance that by Díez et al. (1995) for resistant tomato cultivars in Spain. In recent years, TSWresistant tomato cultivars have become standard for most commercial tomato production acreage in Georgia.

\section{Literature Cited}

Aramburu, J. and M. Marti. 2003. The occurrence in north-east Spain of a variant of tomato spotted wilt virus (TSWV) that breaks resistance in tomato (Lycopersicon esculentum) containing the Sw-5 gene. Plant Pathol. 52:407.

Aramburu, J. and M. Rodriguez. 1999. Evaluation of commercial Lycopersicon esculentum hybrids for resistance to tomato spotted wilt tospovirus (TSWV) in Spain. J. Hort. Sci. Biotechnol. 74:743-747.

Best, R.J. 1968. Tomato spotted wilt virus, p. 65146. In: Smith, K.M. and M.A. Lauffer (eds.). Advances in virus res. Vol. 13. Academic Press, New York, NY.

Boiteux, L.S., and L.B. de Giordano. 1993. Genetic basis of resistance against two Tospovirus species in tomato (Lycopersicon esculentum). Euphytica 71:151-154.

Brown, S.L. and J.E. Brown. 1992. Effect of plastic mulch color and insecticides on thrips populations and damage to tomato. HortTechnology 2:208-211.

Bullock, R.C., P.J. Stoffella, C.A. Powell, J.K. Brecht, H.J. McAuslane, and R.K. Yokomi. 1998. Tomato irregular ripening symptom development and ripening of silverleaf whiteflyinfested dwarf cherry tomatoes. J. Amer. Soc. Hort. Sci. 123:119-125.

Canady, M.A., M.R. Stevens, M.S. Barineau, and J.W. Scott. 2001. Tomato spotted wilt virus (TSWV) resistance in tomato derived from Lycopersicon chilense Dun. LA 1938. Euphytica 117:19-25.

Cho, J.J., R.F.L. Mau, T.L. German, R.W. Hartmann, L.S. Yudin, D. Gonsalves, and R. Provvidenti. 1989. A multidisciplinary approach to management of tomato spotted wilt virus in Hawaii. Plant Dis. 62:264-272.

Ciuffo, M., M.M. Finetti-Sialer, D. Gallitelli, and M. Turina. 2005. First report in Italy of a resistance-breaking strain of Tomato spotted wilt virus infecting tomato cultivars carrying the Sw5 resistance gene. Plant Pathol. 54:564.

de Haan, P., T. Ultzen, M. Prins, J. Gielen, R. Goldbach, and M. van Grinsven. 1996. Transgenic tomato hybrids resistant to tomato spotted wilt virus infection. Acta Hort. 320:306-315.

used in the crossing of these two hybrids. Further work needs to be done to determine if heterozygous resistance from $S w-7$ is not as effective as that of $S w-5$.

We observed no detectable field resistance to the thrips vectors in the TSWresistant tomato lines evaluated. Fortunately, TSW-resistant cultivars are amenable with the use of thrips-reducing tactics such as metallic reflective mulch and insecticide treatments (Riley and Pappu, 2004). Although there are other TSW-resistant tomato lines to evaluate, e.g., 'Viradoro', a resistant cultivar from Brazil (Giordano et al., 2000), this study provides extensive field data on commonly available resistant cultivars, unlike the greenhouse evaluations of Mitidieri et al. (2001). Also, these studies clearly demonstrated the use of commercially available TSW-resistant cultivars in the management of this disease under field conditions in the southeastern United States, similar to the results obtained
Díez, M.J., S. Roselló, C. Jordá, A. Lacasa, J. Costa, and F. Nuez. 1995. Agronomic behaviour of tomato cultivars and lines resistant to TSWV and influence of inoculation methods. Acta Hort. 412:527-532.

Edwardson, J.R. and R.G. Christie. 1986. Tomato spotted wilt virus, p. 563-580. Viruses infecting forage legumes. Vol. III. University of Florida, Gainesville, FL.

Giordano, L.B., A.C. de A'vila, J.M. Charchar, L.S. Boiteux, and E. Ferraz. 2000. 'Viradoro': A Tospovirus-resistant processing tomato cultivar adapted to tropical environments. HortScience 35:1368-1370. etables. Tospoviruses in Solanaceae and other crops in the coastal plain of Georgia. College of Agricultural and Environmental Sciences Bulletin 1354. p. 24-27.

Goldbach, R. and D. Peters. 1994. Possible causes of the emergence of tospovirus diseases. Semin. Virol. 5:113-120.
Gitaitis, R. 2009. . Tospoviruses in Georgia veg- 
Gordillo, L.F., M.R. Stevens, M.A. Millard, and B. Geary. 2008. Screening two Lycopersicon peruvianum collections for resistance to Tomato spotted wilt virus. Plant Dis. 92:694-704.

Greenough, D.R., L.L. Black, and W.P. Bond. 1990. Aluminum-surfaced mulch: An approach to the control of tomato spotted wilt virus in solanaceous crops. Plant Dis. 74:805-808.

Groves, R.L., J.F. Walgenbach, J.W. Moyer, and G.G. Kennedy. 2001. Overwintering of Frankliniella fusca (Thysanoptera: Thripidae) on winter annual weeds infected with Tomato spotted wilt virus and patterns of virus movement between susceptible weed hosts. Phytopathology 91:891-899.

Groves, R.L., J.F. Walgenbach, J.W. Moyer, and G.G. Kennedy. 2002. The role of weed hosts and tobacco thrips, Frankliniella fusca, in the epidemiology of Tomato spotted wilt virus. Plant Dis. 86:573-582.

Iizuka, N., L.S. Boiteux, L.B. de Giordano, T. Nagata, and A.N. Dusi. 1993. Sources of resistance to tomato spotted wilt virus (TSWV) in wild Lycopersicon species. Tomato Genet. Coop. Rep. 43:20-22.

Joost, P.H. and D.G. Riley. 2004. Sampling techniques for thrips (Thysanoptera: Thripidae) in pre-flowering tomato. J. Econ. Entomol. 97: 1450-1454.

Krishna, K.N.K., D.E. Ullman, and J.J. Cho. 1993. Evaluation of Lycopersicon germplasm for tomato spotted wilt tospovirus resistance by mechanical and thrips transmission. Plant Dis. 77:938-941.

Kumar, N.K.K., D.E. Ullman, and J.J. Cho. 1995 Resistance among Lycopersicon species to Frankliniella occidentalis (Thysanoptera: Thripidae). J. Econ. Entomol. 88:1057-1065.

Latham, L.J. and R.A.C. Jones. 1998. Selection of resistance breaking strains of Tomato spotted wilt tospovirus. Ann. Appl. Biol. 133:385-402.
Mandal, B., H.R. Pappu, A.S. Csinos, and A.K. Culbreath. 2006. Response of peanut, pepper, tobacco, and tomato cultivars to two biologically distinct isolates of Tomato spotted wilt virus. Plant Dis. 90:1150-1155.

Mitidieri, M., E. dal Bo, J.A. Fernandez, P.F Martinez, and N. Castilla. 2001. Evaluation of tomato hybrids resistant to TSWV under greenhouse conditions in Argentina. Acta Hort. 559: 775-779.

Oetting, R.D., R.J. Beshear, T.-X. Liu, S.K. Braman, and J.R. Baker. 1993. Biology and identification of thrips on greenhouse ornamentals. Georgia Agricultural Experiment Station Research Bulletin 414

Olson, S.M. 2009. Physiological, nutritional, and other disorders of tomato fruit. HS-954, University of Florida, Gainesville, FL.

Persley, D.M., J.E. Thomas, and M. Sharman 2006. Tospoviruses-An Australian perspective. Australas. Plant Pathol. 35:161-180.

Pico, B., J. Herraiz, J.J. Ruiz, and F. Nuez. 2002. Widening the genetic basis of virus resistance in tomato. Sci. Hort. 94:73-89.

Reitz, S.R., E.L. Yearby, J.E. Funderburk, J. Stavisky, M.T. Momol, and S.M. Olson. 2003. Integrated management tactics for Frankliniella thrips (Thysanoptera: Thripidae) in field-grown peppers. J. Econ. Entomol. 96:1201-1214.

Riley, D. and H. Pappu. 2004. Tactics for management of thrips (Thysanoptera: Thripidae) and Tomato Spotted Wilt Tospovirus in tomato. J. Econ. Entomol. 97:1648-1658.

Riley, D.G. and H. Pappu. 2000. Evaluation of tactics for management of thrips-vectored tomato spotted wilt Tospovirus in tomato. Plant Dis. 84:847-852.

Saidi, M. and S.D. Warade. 2008. Tomato breeding for resistance to Tomato spotted wilt virus (TSWV): An overview of conventional and molecular approaches. Czech J. Genet. Plant Breed. 44:83-92.

Sargent, S.A. and C.L. Moretti. 2004. The commercial storage of fruits, vegetables, and florist and nursery stocks: Tomato. USDA, ARS Agriculture handbook 66. 16 Nov. 2011. <http:// www.ba.ars.usda.gov/hb66/138tomato.pdf $>$.

SAS Institute. 2003. User's manual, version 9.1. SAS Institute, Cary, NC.

Stannard, L.J. 1968. The thrips, or thysanoptera, of Illinois. Illinois Natural History Survey Bulletin 29:215-552.

Stevens, M.R., E.M. Lamb, and D.D. Rhoads. 1995. Mapping the $S w-5$ locus for tomato spotted wilt virus resistance in tomatoes using RADP and RFLP analyses. Theor. Appl. Genet. 90:451456.

Stevens, M.R., S.J. Scott, and R.C. Gergerich. 1992. Inheritance of a gene for resistance to tomato spotted wilt virus (TSWV) from Lycopersicon peruvianum Mill. Euphytica 59:9-17.

Stevens, M.R., S.J. Scott, and R.C. Gergerich. 1994. Evaluation of seven Lycopersicon species for resistance to Tomato spotted wilt virus (TSWV). Euphytica 80:79-84.

Stevens, M.R., J.W. Scott, B.D. Geary, J.J. Cho, L.F. Gordillo, D.M. Persley, F.D. Memmott, and J.J. Stevens. 2006. Current status of resistance to Tospoviruses in tomato. Tampa, FL, 7-11 May 2006 (Tomato Breeders Roundtable and Tomato Quality Workshop abstr. 34).

Ullman, D.E., J.L. Sherwood, and T.L. German. 1997. Thrips as vectors of plant pathogens, $p$. 539-565. In: Lewis, T. (ed.). Thrips as a crop pests. CAB International, Wallingford, UK.

Yudin, L.S., J.J. Cho, and W.C. Mitchell. 1986. Host range of western flower thrips, Frankliniella occidentalis (Thysanoptera: Thripidae), with special reference to Leucaena glauca. Environ. Entomol. 15:1292-1295. 